\title{
Nanobeam Scanning Diffraction for Orientation Mapping of Polymers
}

Karen C. Bustillo ${ }^{1}$, Ouliana Panova ${ }^{2,3}$, X. Chelsea Chen ${ }^{3,4}$, Christopher J. Takacs ${ }^{5}$, Jim Ciston ${ }^{1}$, Colin Ophus ${ }^{1}$, Nitash P. Balsara ${ }^{3,4}$, and Andrew M. Minor ${ }^{1,2,3}$.

1. National Center for Electron Microscopy, Molecular Foundry, Lawrence Berkeley National

Laboratory, Berkeley, USA.

2. Department of Materials Science and Engineering, University of California, Berkeley, USA.

${ }^{3}$ Materials Sciences Division, Lawrence Berkeley National Laboratory, Berkeley, USA.

${ }^{4}$ Department of Chemical and Biomolecular Engineering, University of California, Berkeley, USA.

5. Photon Sciences, Stanford University, Menlo Park, USA.

The technique of nanobeam scanning diffraction in the transmission electron microscope (TEM)[1] is applied to beam sensitive organic small-molecule and polymer thin films with the goal of producing a map of the crystalline regions and their crystallographic orientations. Many organic thin films have anisotropic transport and mechanical properties. Correlating the nanostructure of the material with these properties, as well as understanding the synthesis pathways to achieve a specific nanostructure motivate the desire to image these materials with nanometer resolution. Yet organic materials are typically electron beam sensitive and made from low $\mathrm{Z}$ elements that produce little contrast in the TEM image. If the polymer has crystalline regions or aligned moities, diffraction from these ordered domains can create contrast in the image provided that the diffraction can be captured before the electron beam destroys the order.

Here we present data from a semiconducting polymer system, a small molecule material, and an ionic conducting polymer to illustrate the generality and flexibility of the approach. The first example is poly3 hexithiophene (P3HT) an organic semiconductor [2]. The second example is a small molecule material of 7,7'-(4,4-bis(2-ethylhexyl)-4H- silolo[3,2-b:4,5-b']dithiophene-2,6-diyl)bis(6-fluoro-4-(5'-hexyl-[2,2'bithiophen]-5-yl)benzo[c][1,2,5]thiadiazole), $\left(p\right.$-DTS $\left.\left(\mathrm{FBTTh}_{2}\right)_{2}\right) \quad[3,4] . \quad$ The third example is polyethylene oxide [5].

The scanning diffraction experiment uses a convergent electron probe in scanning mode to step across the region of interest and acquire a diffraction pattern image at each step. Because each $\mathrm{x}, \mathrm{y}$ position in real space has an associated $\mathrm{k}_{\mathrm{x}} \mathrm{x} \mathrm{k}_{\mathrm{y}}$ image, a 4-dimensional dataset is acquired. By applying virtual apertures to the dataset one can reconstruct a real space image corresponding to a selected diffraction condition. Assigning a color to each reflection allows one to build an orientation map of the sample in real space with the spatial resolution defined by the scanning step size (Figure 1).

We review the experimental parameters that optimize the scanning diffraction experiment for beam sensitive materials. The challenge is to obtain sufficient $S: N$ in the diffraction patterns such that a computer algorithm can find and identify the location of the spots with few errors. Keeping the sample at liquid nitrogen temperature during the experiment extends the duration of the diffraction as it minimizes the damage due to the electron beam. Fast direct electron detectors improve S:N by capturing the diffracted image before the damage adds noise onto the detector. The shape of the probe and total dose must be carefully controlled and optimized for a given real space step size.

The large datasets generated by the scanning nanobeam diffraction method necessitate optimized 
computer algorithms for data analysis. Code to do this analysis has been developed [6], but some algorithms specific to the polymer dataset have been added to the general analysis workflow. We present data from an uncorrected FEI Titan 80-300kVwith a Gatan Orius 830 CCD and an aberration corrected FEI TEAM I microscope outfitted with a Gatan K2-IS direct electron detector.

By using both liquid nitrogen sample temperature and a direct electron detector we are able to achieve $<5 \mathrm{~nm}$ spatial resolution in an orientation map of a beam sensitive polymer. These results indicate that nanobeam scanning diffraction has the potential to be a powerful tool for imaging the nanostructure of beam sensitive polymers.

References:

[1] C Gammer et al, Ultramicroscopy 155 (2015), p. 1.

[2] O Panova et al, Micron 88 (2016), p. 30.

[3] O Panova et al, manuscript in preparation.

[4] T Van der Poll et al, Advanced Materials 24 (2012), p. 3646.

[5] F Croce et al, Nature 394 (1998), p. 456.

[6] T Pekin et al, Ultramicroscopy, in press.

[7] Work at the Molecular Foundry was supported by the Office of Science, Office of Basic Energy Sciences, of the U.S. Department of Energy under Contract No. DE-AC02-05CH11231. OP, XCC, NPB and AMM acknowledge the Electron Microscopy of Soft Matter Program from the Office of Science, Office of Basic Energy Sciences, Materials Sciences and Engineering Division of the U. S. Department of Energy under Contract No. DE-AC02-05CH11231.

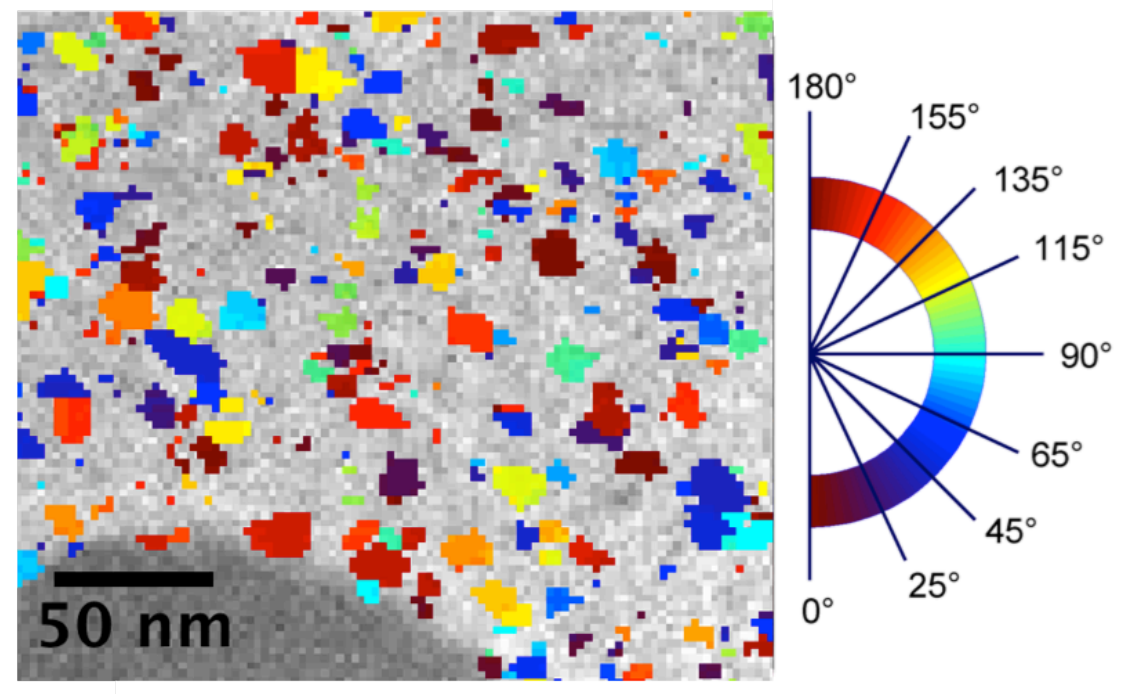

Figure 1. Real space orientation map constructed from a 4D dataset of poly-3 hexithiophene (P3HT), an organic semiconductor. Spatial resolution is defined by the STEM pixel size of $4.9 \mathrm{~nm}$. The color corresponds to the orientation of the $\pi-\pi$ stacking diffraction reflection as shown by the color legend on the right. Diffraction images were acquired at $300 \mathrm{kV}$ using a $0.5 \mathrm{mrad}$ convergent probe with a probe size of 3-5 $\mathrm{nm}$ and an exposure time of $2.5 \mathrm{~ms}$ per diffraction pattern. 